University of South Carolina

Scholar Commons

$5-7-2014$

\title{
Characterization of 3D Interconnected Microstructural Network in Mixed Ionic and Electronic Conducting Ceramic Composites
}

\author{
William M. Harris
}

Kyle S. Brinkman

Ye Lin

University of South Carolina - Columbia, linye@mailbox.sc.edu

Dong Su

Alex P. Cocco

See next page for additional authors

Follow this and additional works at: https://scholarcommons.sc.edu/emec_facpub

Part of the Applied Mechanics Commons, Ceramic Materials Commons, and the Electro-Mechanical Systems Commons

\section{Publication Info}

Published in Nanoscale, Volume 6, Issue 9, 2014, pages 4480-4485.

CNanoscale 2014, Royal Society of Chemistry.

This article cannot be redistributed or further made available.

This article was first published by the Royal Society of Chemistry and can be found at http://dx.doi.org/ 10.1039/c3nr06684c

Harris, W. M., Brinkman, K. S., Lin, Y., Su, D., Cocco, A. P., Nakajo, A., DeGostin, M. B., Chen-Wiegart, Y.-C. K., Wang, J., Chen, F., Chu, Y. S., \& Chiu, W. K. (7 May 2014). Characterization of 3D Interconnected Microstructural Network in Mixed Ionic and Electronic Conducting Ceramic Composites. Nanoscale, 6 (9), 4480 - 4485. http://dx.doi.org/10.1039/C3NR06684C

This Article is brought to you by the Mechanical Engineering, Department of at Scholar Commons. It has been accepted for inclusion in Faculty Publications by an authorized administrator of Scholar Commons. For more information, please contact digres@mailbox.sc.edu. 


\section{Author(s)}

William M. Harris, Kyle S. Brinkman, Ye Lin, Dong Su, Alex P. Cocco, Arata Nakajo, Matthew B. DeGostin, Yu-chen Karen Chen-Wiegart, Jun Wang, Fanglin Chen, Yong S. Chu, and Wilson K. S. Chiu 


\section{Characterization of 3D interconnected microstructural network in mixed ionic and electronic conducting ceramic composites $\uparrow$}

Cite this: Nanoscale, 2014, 6, 4480

Received 17th December 2013 Accepted 26th February 2014

DOI: $10.1039 / c 3 n r 06684 c$
William M. Harris, $\neq^{\mathrm{ab}}$ Kyle S. Brinkman, $\ddagger^{\mathrm{ac}}$ Ye Lin, ${ }^{\text {af }}$ Dong Su, ${ }^{\mathrm{d}}$ Alex P. Cocco, ${ }^{\text {ab }}$ Arata Nakajo, ${ }^{a b}$ Matthew B. DeGostin, ${ }^{\text {ab }}$ Yu-chen Karen Chen-Wiegart, ${ }^{e}$ Jun Wang, ${ }^{e}$ Fanglin Chen, ${ }^{\text {af }}$ Yong S. Chu ${ }^{g}$ and Wilson K. S. Chiu ${ }^{\star a b}$

www.rsc.org/nanoscale

The microstructure and connectivity of the ionic and electronic conductive phases in composite ceramic membranes are directly related to device performance. Transmission electron microscopy (TEM) including chemical mapping combined with X-ray nanotomography (XNT) have been used to characterize the composition and 3-D microstructure of a MIEC composite model system consisting of a $\mathrm{Ce}_{0.8} \mathrm{Gd}_{0.2} \mathrm{O}_{2}(\mathrm{GDC})$ oxygen ion conductive phase and a $\mathrm{CoFe}{ }_{2} \mathrm{O}_{4}$ (CFO) electronic conductive phase. The microstructural data is discussed, including the composition and distribution of an emergent phase which takes the form of isolated and distinct regions. Performance implications are considered with regards to the design of new material systems which evolve under non-equilibrium operating conditions.

Ceramic membranes which transport oxygen ions play an essential role in a number of energy conversion systems including solid oxide fuel cells (SOFC) ${ }^{1}$ Li-air batteries, ${ }^{2}$ oxygen separation and permeation membranes for oxygen production,,$^{3-5}$ partial oxidation of methane ${ }^{6,7}$ and clean coal production via oxy-combustion resulting in significant reductions in $\mathrm{CO}_{2}$ emissions from coal fired power plants. ${ }^{8,9}$ In addition to

${ }^{a}$ HeteroFoaM Center, a DOE Energy Frontier Research Center, USA. E-mail: wchiu@ engr.uconn.edu

${ }^{b}$ Department of Mechanical Engineering, University of Connecticut, Storrs, CT 06269, USA

${ }^{c}$ Department of Materials Science and Engineering, Clemson University, Clemson, SC 29634, USA

${ }^{d}$ Center for Functional Nanomaterials, Brookhaven National Laboratory, Upton, NY 11973, USA

${ }^{e}$ Photon Sciences Directorate, Brookhaven National Laboratory, Upton, New York 11973, USA

${ }^{f}$ Department of Mechanical Engineering, University of South Carolina, Columbia, SC 29208, USA

${ }^{g}$ National Synchrotron Light Source II, Brookhaven National Laboratory, Upton, NY 11973, USA

† Electronic supplementary information (ESI) available. See DOI: 10.1039/c3nr06684c

\$ These authors contributed equally to this work. energy generation systems, ceramic membranes have the potential to be increasingly prominent in advanced manufacturing processes including electrochemical reduction cells for metals processing ${ }^{\mathbf{1 0 , 1 1}}$ and spent nuclear fuel re-processing $^{12}$ as well as high temperature electrolysis cells for hydrogen production ${ }^{\mathbf{1 3}}$ and state of the art combustion control sensors. ${ }^{14}$

The properties and function of these devices are controlled by mixed ionic-electronic conductivity (MIEC) in the respective material system. MIEC can be achieved by either (i) selecting a material which supports both ionic and electronic conduction or (ii) forming a two phase composite of an ionic conductor and an electronic conductor with the appropriate microstructure consisting of two percolated networks. The present work focuses on the latter strategy for tailoring MIEC, namely fabricating a composite of ionic and electronic conductive materials. The inherent flexibility of multiphase systems makes it more feasible to tune the various properties needed to satisfy the set of stringent chemical, electrical, thermal and mechanical properties for a variety of MIEC applications. In this work, $\mathrm{Ce}_{0.8} \mathrm{Gd}_{0.2} \mathrm{O}_{2}$ (GDC) was chosen as the oxygen ion-conducting phase due to its excellent ionic conductivity near $0.1 \mathrm{~S} \mathrm{~cm}^{-1}$ at $800{ }^{\circ} \mathrm{C}^{15}$ The chosen electronic conductor is $\mathrm{CoFe}_{2} \mathrm{O}_{4}$ (CFO) due to its stable spinel structure and electronic conductivity greater than $1 \mathrm{~S} \mathrm{~cm}^{-1}$ at $800{ }^{\circ} \mathrm{C} .^{\mathbf{1 6 , 1 7}}$ This system has previously demonstrated high oxygen flux and stability under reducing environments in the presence of $\mathrm{CO} / \mathrm{CO}_{2}$, and serves as a model system for general composite membrane studies., ${ }^{\mathbf{4 , 1 6 , 1 8 , 1 9}}$

It is recognized that the performance of ceramic composites are not necessarily bounded by the properties of the individual constituents; the interaction of these phases including interfacial contributions and phase evolution may result in a global response different than the properties expected from simple mixture rules. ${ }^{\mathbf{2 0}}$ The emergent properties arising from these interactions at the mesoscale present new opportunities, as well as challenges, for materials performance and functionality. ${ }^{21} \mathrm{In}$ MIEC ceramic membranes composites, reactions between the constituent phases may result in additional interfacial or bulk 
phase formation that impact ionic and electronic transport. For example, Kharton in 2001 reported an order of magnitude drop in the ionic conductivity of a $\mathrm{Ce}_{0.8} \mathrm{Gd}_{0.2} \mathrm{O}_{2}-\mathrm{La}_{0.7} \mathrm{Sr}_{0.3} \mathrm{MnO}_{3}$ (GDC-LSM) composite membrane after elevated temperatures and longer sintering times were used for membrane fabrication. ${ }^{22}$ This behavior was primarily attributed to the formation of resistive "blocking" layers at GDC-LSM interfaces inhibiting ionic transport. An additional effect to consider was changes in stoichiometry due to the potential diffusion of $\mathrm{La}^{3+}$ and $\mathrm{Sr}^{3+}$ into the GDC lattice, resulting in the formation of defect associates between oxygen vacancies and dopant cations and thus reducing ionic conductivity. In the GDC-CFO system there is currently limited understanding of the impact of these interfacial phases on system performance. ${ }^{16}$ In addition, unintentional formation of new phases in a bulk manner (whether it be during material processing or operation), on the same length scale as the primary phases, may also have impacts on the membrane performance, particularly from a transport perspective.

Recently, synchrotron based 3-D tomography has been used to provide elemental and chemical state specific information for $\mathrm{NiO} / \mathrm{Li}$ battery electrodes and track changes occurring in $\mathrm{Fe}_{2} \mathrm{O}_{3}-$ $\mathrm{Fe}_{2} \mathrm{TiO}_{5}$ catalyst as well as explore the mechanism of reductionoxidation cycling of nickel based oxides in solid oxide fuel cells. $^{23-25}$ This technique addresses unexplored issues at the mesoscale; how do the phases, interfaces, resulting microstructure and potential changes during operation effect performance? Here we demonstrate the use of this technique to identify and map distinct phases in MIEC ceramic composite membranes. Ultimately, this 3-D microstructural data with varying volume fraction of mixtures and microstructures will be incorporated into computer based simulations in order to predict and design new material systems with emergent properties as well as predict the performance of current material systems which evolve under non-equilibrium operating conditions.

The composite membranes were prepared by mixing the respective volume percent of the ionic conductive phase $\mathrm{Ce}_{0.8} \mathrm{Gd}_{0.2} \mathrm{O}_{2-\delta}$ (InfraMat Advanced Materials LLC) and the electronic conductive phase $\mathrm{CoFe}_{2} \mathrm{O}_{4}$ (InfraMat Advanced Materials LLC) by ball milling powder in ethanol for 6 hours. The composition was fixed at 60 volume percent GDC and 40 volume percent CFO (60GDC-40CFO) in the present study. After drying and sieving, ceramics were mixed with a binder and uniaxially pressed into pellets of $16 \mathrm{~mm}$ diameter with a force of $20 \mathrm{kN}$ and sintered in air at $1300{ }^{\circ} \mathrm{C}$ for 2 hours. The sintering temperature was selected as a compromise between the typical temperatures used for GDC and CFO sintering which are about $1500{ }^{\circ} \mathrm{C}$ and $1000{ }^{\circ} \mathrm{C}$, respectively. ${ }^{26} \mathrm{~A}$ ramp rate of $2{ }^{\circ} \mathrm{C}$ per minute was used during heating and cooling stages.

In order to confirm the chemical phases present and the spatial resolution required for X-ray nanotomography (XNT) experiments, MIEC starting materials and ceramic membranes were characterized by High Resolution Transmission Electron Microscopy (HRTEM). Samples of the GDC and CFO powders were prepared for electron microscopy by supersonic dispersion followed by careful dipping onto a Holey Carbon 400 mesh $\mathrm{Cu}$ grid (Ted Pella, Inc.) The sintered GDC-CFO membranes were prepared by a classic dimpling method using a Gatan dimpler 626 with the final sample thickness reduced to less than $10 \mathrm{~nm}$ accomplished by ion milling (Fischione Model 1010). Scanning Transmission Electron Microscopy (STEM) with high-angle annular dark-field imaging (HAADF) and Energy Dispersive X-ray (EDX) mapping (with scan speed $\sim 0.6 \mathrm{~nm} \mathrm{~s}^{-1}$ ) were performed to distinguish each constituent phase in the composite. Bright field (BF) images and selected area electron diffraction (SAED) patterns were acquired using a JEOL-2100F TEM equipped with a Schottky field-emission gun (FEG), with Cs = $1.0 \mathrm{~mm}$ operated at $200 \mathrm{kV}$.

Electron microscopy of the GDC and CFO starting powders is shown in Fig. 1. As shown in Fig. 1a, the individual nano-sized GDC powder is in the range of 12-50 $\mathrm{nm}$. The SAED (Fig. 1b) can be indexed to GDC (ICSD \#28796), with cubic symmetry and lattice constant of $a=5.42 \AA$, with no other secondary phases observed. The BF image of CFO in Fig. 1c shows mainly two different size distributions of particles which are labeled by circles (A) and (B). Particles in the (A) area are smaller size particles in the 5-10 nm range, while larger particles (100$250 \mathrm{~nm}$ ) are found in (B) area phases. The SAED pattern on the CFO powder, shown in (d), was well indexed to ICSD \#109045 as $\mathrm{CoFe}_{2} \mathrm{O}_{4}$ exhibiting a cubic structure with $a=8.39 \AA$ and a space group of $F d \overline{3} m$ (227).

The elements and phase distribution of the sintered 60GDC40CFO composite were discriminated using Z-contrast imaging, generated by HAADF-STEM and EDX as shown in Fig. 2. Three different contrast levels (bright, grey and dark) could be clearly found based on the HAADF image (Fig. 2a), indicating different average $Z$ values of three solid phases. The EDX mapping in Fig. 2b-e could further be used to help distinguish the phases'

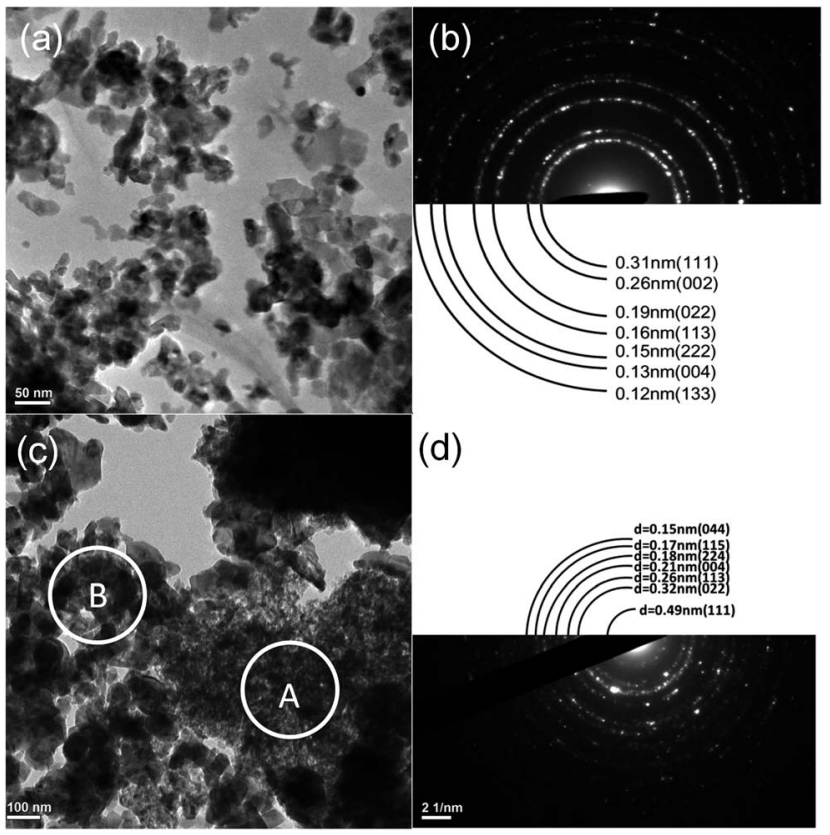

Fig. 1 (a) BF image and (b) SAED of GDC powder. (c) BF image and (d) SAED of CFO powder. 


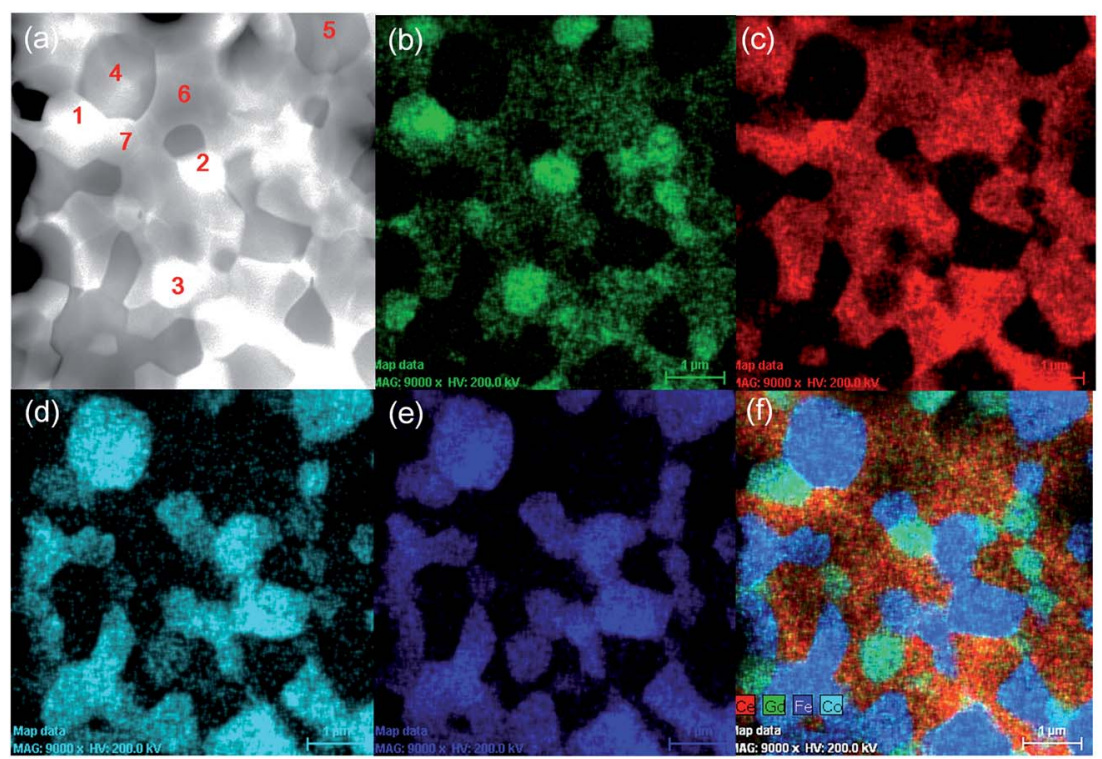

Fig. 2 (a) HAADF image for the GDC-CFO (60\% GDC, 40\% CFO by volume) sample. (b-e) STEM elemental mapping for Gd (green), Ce (red), Co (cyan) and Fe (blue) respectively. (f) Composite elemental map of $\mathrm{Gd}, \mathrm{Ce}, \mathrm{Co}$, and $\mathrm{Fe}$.

constituent elements. The primary phases of GDC and CFO contain $\mathrm{Gd} / \mathrm{Ce}$ and $\mathrm{Co} / \mathrm{Fe}$ respectively, as expected, corresponding to the grey and dark grains in Fig. 2a respectively. In addition to these two phases, a brightest and heaviest phase seen in Fig. 2a is an emergent phase, which can be seen in the EDX maps to contain Gd, Ce, Co and Fe. More substantial quantitative analysis in the ESI $\uparrow$ suggests a chemical formula of $\mathrm{Gd}_{0.374} \mathrm{Ce}_{0.079} \mathrm{Co}_{0.077} \mathrm{Fe}_{0.47} \mathrm{O}_{x}$, but for simplicity the phase will be denoted as GFCC. The electron energy loss spectroscopy (EELS) scan taken inside the GFCC grains (Fig. S2 $\dagger$ ) also show the "fingerprints" for all four elements. In addition, this will be verified using a 3-D imaging method in the following section.

Although advantageous in terms of its chemical mapping capabilities and spatial resolution, the electron microscopy imaging and elemental mapping results could only produce a 2D description of the complex microstructure of the composite material. To achieve a truly $3 \mathrm{D}$ quantitative description of the composite, the 3-D microstructure of the material was measured using X-ray nanotomography (XNT), which was performed on the transmission X-ray microscope (TXM) at the National Synchrotron Light Source, beamline X8C. ${ }^{27}$ Energy tuning capability of synchrotron X-rays, employed in XNT measurements, allowed imaging of 3D distributions of the different material phases identified in the electron microscopy studies. Element-sensitive XNT measurements were performed at X-ray beam energies above and below elemental absorption edges, in order to invoke substantial elemental contrast in the absorption images. ${ }^{25,28}$ For the materials in this study, tomographic imaging spanning the Fe K edge $(7112 \mathrm{eV})$ and $\mathrm{Gd} \mathrm{L}_{3}$ edge $(7243 \mathrm{eV})$ was performed by imaging at 7090,7200 , and $7280 \mathrm{eV}$, as shown in Fig. 3. Approximations of X-ray absorption behavior shown in Fig. 3 suggest distinguishing the expected phases by the following criteria: ${ }^{29}$
- GDC: no change across Fe $\mathrm{K}$ edge, very small increase across the $\mathrm{Gd} \mathrm{L}_{3}$ edge.

- CFO: increase in attenuation across Fe K-edge, flat across $\mathrm{Gd} \mathrm{L}_{3}$ edge.

- GFCC: attenuation increases across both $\mathrm{Fe} \mathrm{K}$ - and $\mathrm{Gd} \mathrm{L}_{3}$ edges.

- Pore: negligible attenuation at all energy levels.

The sample used for the XNT measurements was prepared using a focused ion beam-scanning electron microscope..$^{30}$ The ion beam was used to mill a cylindrical sample approximately 10 microns in diameter and height. A micromanipulator probe was used to lift the sample from the bulk material and move it

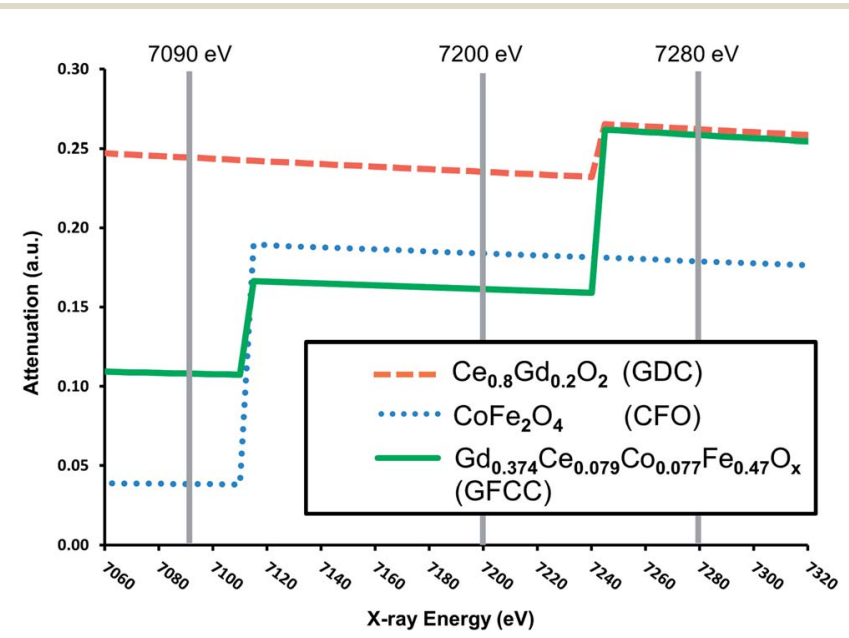

Fig. 3 Density-based approximations show $X$-ray absorption behavior spanning the Fe $\mathrm{K}$ edge (7112 eV) and the $\mathrm{Gd} \mathrm{L}_{3}$ edge $(7243 \mathrm{eV})$. XNT imaging was performed at 7090, 7200, and $7280 \mathrm{eV}$ to span both absorption edges and identify the solid phases based on their contrast change across each edge. 


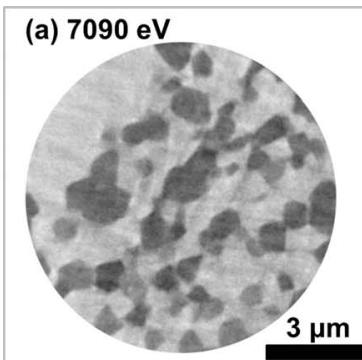

(d) $7200-7090$

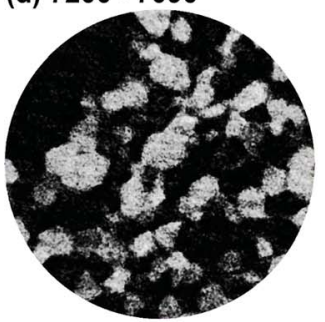

(b) $7200 \mathrm{eV}$

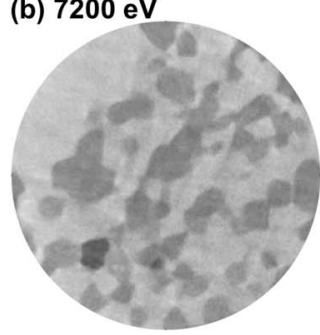

(e) $7280-7200$

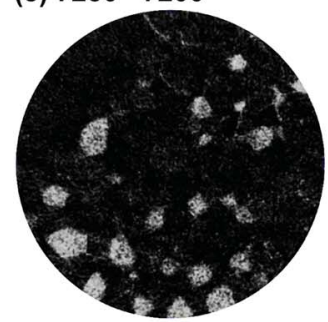

(c) $7280 \mathrm{eV}$

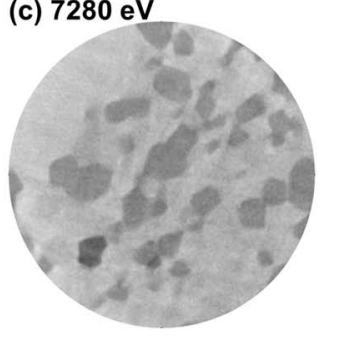

(f)

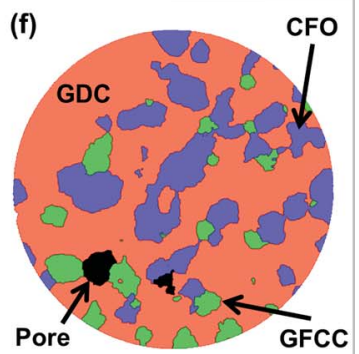

Fig. 4 X-ray nanotomography imaging of GDC-CFO. (a), (b), and (c) cross sectional slices of the nanotomography data obtained at each energy level (Fig. 3). (d) Absorption contrast across the Fe K edge. (e) Absorption contrast across the Gd $\mathrm{L}_{3}$ edge. (f) Segmented, labeled structure.

to the tip of a stainless steel pin, to which it was attached with platinum deposition.

The sample was then mounted in the TXM at NSLS, with an $\mathrm{X}$-ray zoneplate providing $30 \mathrm{~nm}$ spatial resolution. For each energy level (7090 eV, $7200 \mathrm{eV}$, and $7280 \mathrm{eV}$ ), tomography was performed by rotating the sample over a range of 180 degrees at 0.25 degree increments. At each angle, a projection image was collected with an exposure time of 20 seconds and a camera binning value of 2 (giving an image pixel size of $19.5 \mathrm{~nm}$ ). The camera position was adjusted between each data set to maintain a consistent magnification of the sample. Each tomographic data set was reconstructed using a filtered back projection algorithm to generate three 3-D volumes. Crosssectional slices representative of each energy level are shown in Fig. 4a-c.

Minor pixilation, imaging artifacts, and outlier pixels were removed from each data set using a 3-D median filter, which replaces each voxel with the median of its value and its six nearest neighbors. Subtraction of the volumes spanning the Fe $\mathrm{K}$ edge (Fig. 4d, 7200-7090 eV) and the Gd $\mathrm{L}_{3}$ edge (Fig. 4e, 7280-7200 eV) was performed on a voxel-by-voxel basis to highlight the attenuation changes across the absorption edges. Using the contrast, regions belonging to each phase could be identified based on the listed criteria. In addition, a maximum gradient magnitude map was created by taking the maximum of the gradient of Fig. 4a-c on a voxel-by-voxel basis. The identified phase regions and the gradient map were used as inputs to a watershed algorithm (with the identified regions serving as seeds, and the gradient map defining phase boundaries) to create a segmented structure, as shown in Fig. $4 \mathrm{f}$.

Although cross-sectional 2-D slices are shown in Fig. 4 for demonstration purposes, the entire process was conducted in 3-D. Several orthographic views of the 3-D structure are shown in Fig. 5a, and an animation of the segmented sample is available online as ESI. $\uparrow$ The 3-D structure was used to compute size distributions, volume fraction, tortuosity, and contiguity of each phase. Details of these calculations are provided in the ref. 31 and 32. The size distributions of each phase are shown in Fig. 5b, and the other properties are reported in Table 1 . The GDC and CFO can both be characterized by a mean "particle" diameter of over 1 micron, whereas the GFCC and pore phases are mostly in the sub-micron range. The mean is evaluated on a volume-weighted basis for each phase, i.e. an equal volume of each phase is contained above and below its characteristic mean. The small size of the pore and GFCC phases helps explain why they form only isolated regions, and would likely not be substantial obstacles to transport.

The spatial variation in the property values was tested by analyzing multiple smaller sub-volumes of the structure. Only minor variations of the properties were observed, indicating that the properties reported in Table 1 are volume-independent. The measured volume fractions of GDC and CFO are similar to the nominal composition of the fabricated membrane $(60 \%$ and
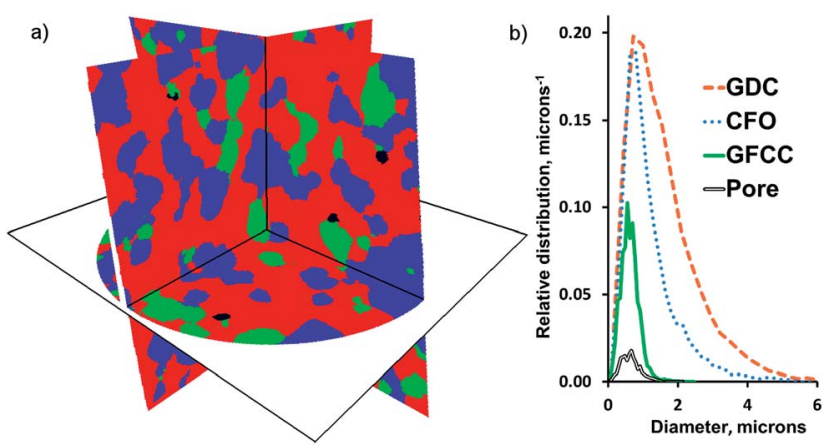

Fig. 5 (a) Orthographic views demonstrate the 3-D structure. (b) Phase size distributions for the full 3-D structure. 
Table 13 -D volume fraction, tortuosity, contiguity, and mean size of each phase

\begin{tabular}{lcclc}
\hline & GDC & CFO & GFCC & Pore \\
\hline Volume fraction (\%) & 57 & 33 & 9 & 1 \\
Contiguity (\%) & 100 & 96 & 0 & 0 \\
Tortuosity & 1.24 & 2.6 & - & - \\
Mean diameter, $\mu \mathrm{m}$ & 1.55 & 1.17 & 0.62 & 0.64 \\
\hline
\end{tabular}

$40 \%$, respectively) with some clear loss of each phase contributing to the formation of the GFCC phase, which accounts for $9 \%$ of the structure, by volume. Pores account for the remaining $1 \%$ of the volume. As such small constituents, the pores and GFCC each consist of completely isolated regions, and form no connected percolating networks through the material. The lack of a contiguous pore structure is important in preventing gas crossover, which would reduce the chemical potential driving force for separation applications. The formation of isolated and distinct GFCC regions may produce such effects as the geometric alteration of transport pathways and change of local stoichiometry in neighboring phases. With the pore and GFCC phases accounting for $10 \%$ of the volume, the GDC and CFO both still form nearly completely connected networks with very few isolated regions, and as such still serve as effective transport pathways with tortuosity values of 1.24 and 2.6 , respectively. Both values of tortuosity are modest, and while the higher tortuosity of CFO does represent a less direct electron transport pathway, the electronic conductivity of CFO is about an order of magnitude higher than the ionic conductivity of GDC, meaning the electron transport geometry is unlikely to be a limiting factor. Considering the implications on bulk transport only, the main impact from the emergent GFCC phase formation is a depletion of Gd from the oxygen ion transport phase GDC. This reduces the $\mathrm{Gd}$ content from 0.2 to $0.16\left(\mathrm{Ce}_{0.8} \mathrm{Gd}_{0.2} \mathrm{O}_{2-\delta} v s\right.$. $\mathrm{Ce}_{0.84} \mathrm{Gd}_{0.16} \mathrm{O}_{2-\delta}$ ), resulting in a slight decrease in the bulk oxygen ion conductivity. ${ }^{15}$ Conversely, the possibility of some appreciable ionic conduction in the emergent GFCC phase and its associated new type of grain boundaries could lessen negative impacts on the membrane transport behavior, and is an area of ongoing investigation. Lastly, the formation of an emergent bulk phase, which is relatively isolated in the microstructure, may be beneficial compared to the possible additional formation of a Gd-rich interface in $\mathrm{CeO}_{2}$ or other interfacial phases, which block ionic transport as has been observed in other MIEC material systems. ${ }^{15,22}$

The resulting 3-D nanostructures obtained from XNT are especially useful for computational studies aimed at predicting material system properties. It is ultimately envisioned that the desired microstructure, volume fraction of individual phases, morphology and interfacial properties will be predicted by computer based simulations instead of trial and error methodologies. The current work outlines a methodology of materials characterization consisting of TEM coupled with XNT for the determination of the 3-D microstructures of the bulk phases in the GDC-CFO model system with a resolution of $\sim 30 \mathrm{~nm}$ as a first step in this process. However, it is realized that engineered interfaces (i.e., grain boundaries) and tailored defects should be a key focus area for the design and fabrication of next generation energy conversion systems including ceramic MIEC composites. This will require more detailed characterization of the interfaces in MIEC composites using HRTEM and higher resolution XNT with resolution greater than $\sim 10 \mathrm{~nm}$ that can potentially probe the interfacial regions resulting in chemical information including tomographic mapping of bulk and grain boundary interfacial regions in polycrystalline materials.

\section{Acknowledgements}

The authors acknowledge financial support from an Energy Frontier Research Center on Science Based Nano-Structure Design and Synthesis of Heterogeneous Functional Materials for Energy Systems (HeteroFoaM Center) funded by the U.S. Department of Energy, Office of Science, Office of Basic Energy Sciences (Award no. DE-SC0001061). Use of the National Synchrotron Light Source, Brookhaven National Laboratory, was supported by the U.S. Department of Energy, Office of Science, Office of Basic Energy Sciences, under contract no. DEAC02-98CH10886. Electron microscopy research has been partially carried out at the Center for Functional Nanomaterials, Brookhaven National Laboratory, which is supported by the U.S. Department of Energy, Office of Basic Energy Sciences, under contract no. DE-AC02-98CH10886.

\section{References}

1 J. Larminie and A. Dicks, Fuel Cell Systems Explained, Wiley, England, 2003.

2 Y. Shao, F. Ding, J. Xiao, J. Zhang, W. Xu, S. Park, J.-G. Zhang, Y. Wang and J. Liu, Adv. Funct. Mater., 2013, 23, 987-1004.

3 P. N. Dyer, R. E. Richards, S. L. Russek and D. M. Taylor, Solid State Ionics, 2000, 134, 21-33.

$4 \mathrm{H}$. Takamura, M. Ogawa, K. Suehiro, H. Takahashi and M. Okada, Solid State Ionics, 2008, 179, 1354-1359.

$5 \mathrm{~K}$. Brinkman, T. Iijima and H. Takamura, Solid State Ionics, 2010, 181, 53-58.

6 H. J. M. Bouwmeester, Catal. Today, 2003, 82, 141-150.

7 I. Kagomiya, T. Iijima, H. Kakuta and H. Takamura, Electrochem. Solid-State Lett., 2005, 8, A70-A73.

8 H. Luo, H. Jiang, T. Klande, Z. Cao, F. Liang, H. Wang and J. Caro, Chem. Mater., 2012, 24, 2148-2154.

9 J. Sunarso, S. Baumann, J. M. Serra, W. A. Meulenberg, S. Liu, Y. S. Lin and J. C. Diniz da Costa, J. Membr. Sci., 2008, 320, 13-41.

10 A. Krishnan, X. G. Lu and U. B. Pal, Metall. Mater. Trans. B, 2005, 36, 463-473.

11 U. B. Pal, D. E. Woolley and G. B. Kenney, JOM, 2001, 53, 3235.

12 L. Burris, D. S. Poa, R. K. Steunenberg and Z. Tomczuk, US Pat. 4,995,948, 1991.

13 C. M. Stoots, J. E. O'Brien, K. G. Condie and J. J. Hartvigsen, Int. J. Hydrogen Energy, 2010, 35, 4861-4870.

14 I.-D. Kim, A. Rothschild and H. L. Tuller, Acta Mater., 2013, 61, 974-1000. 
15 H. Inaba and H. Tagawa, Solid State Ionics, 1996, 83, 1-16.

16 H. Takamura, M. Kawai, K. Okumura, A. Kamegawa and M. Okada, Mater. Res. Soc. Symp. Proc., 2003, 756, 121-126.

17 J.-L. Guo, Y.-D. Chiou, W.-I. Liang, H.-J. Liu, Y.-J. Chen, W.-C. Kuo, C.-Y. Tsai, K.-A. Tsai, H.-H. Kuo, W.-F. Hsieh, J.-Y. Juang, Y.-J. Hsu, H.-J. Lin, C.-T. Chen, X.-P. Liao, B. Shi and Y.-H. Chu, Adv. Mater., 2013, 25, 2040-2044.

18 I. Kagomiya, T. Iijima and H. Takamura, J. Membr. Sci., 2006, 286, 180-184.

19 H. Takamura, Y. Koshino, A. Kamegawa and M. Okada, Solid State Ionics, 2006, 177, 2185-2189.

20 K. L. Reifsnider, W. K. S. Chiu, K. S. Brinkman, Y. Du, A. Nakajo, F. Rabbi and Q. Liu, J. Electrochem. Soc., 2013, 160, F470-F481.

21 G. W. Crabtree and J. L. Sarrao, MRS Bull., 2012, 37, 10791088.

22 V. V. Kharton, A. V. Kovalevsky, A. P. Viskup, F. M. Figueiredo, A. A. Yaremchenko, E. N. Naumovich and F. M. B. Marques, J. Eur. Ceram. Soc., 2001, 21, 1763-1767.

23 F. Meirer, J. Cabana, Y. Liu, A. Mehta, J. C. Andrews and P. Pianetta, J. Synchrotron Radiat., 2011, 18, 773781.
24 F. M. F. de Groot, E. de Smit, M. M. van Schooneveld, L. R. Aramburo and B. M. Weckhuysen, ChemPhysChem, 2010, 11, 951-962.

25 G. J. Nelson, W. M. Harris, J. R. Izzo Jr, K. N. Grew, W. K. S. Chiu, Y. S. Chu, J. Yi, J. C. Andrews, Y. Liu and P. Pianetta, Appl. Phys. Lett., 2011, 98, 173109.

26 Y. M. Abbas, S. A. Mansour, M. H. Ibrahim and S. E. Ali, J. Magn. Magn. Mater., 2011, 323, 2748-2756.

27 J. Wang, Y.-C. Karen Chen, Q. Yuan, A. Tkachuk, C. Erdonmez, B. Hornberger and M. Feser, Appl. Phys. Lett., 2012, 100(14), 143107.

28 K. N. Grew, Y. S. Chu, J. Yi, A. A. Peracchio, J. R. Izzo Jr, Y. Hwu, F. De Carlo and W. K. S. Chiu, J. Electrochem. Soc., 2010, 157, B783-B792.

29 B. L. Henke, E. M. Gullikson and J. C. Davis, At. Data Nucl. Data Tables, 1993, 54, 181-342.

30 J. J. Lombardo, R. Ristau, W. M. Harris and W. K. S. Chiu, J. Synchrotron Radiat., 2012, 19, 789-796.

31 K. N. Grew, A. A. Peracchio, A. S. Joshi, J. R. Izzo Jr. and W. K. S. Chiu, J. Power Sources, 2010, 195, 7930-7942.

32 K. N. Grew, A. A. Peracchio and W. K. S. Chiu, J. Power Sources, 2010, 195, 7943-7958. 